Pacific

Journal of

Mathematics

HARDY'S UNCERTAINTY PRINCIPLE ON SEMISIMPLE GROUPS

M. Cowling, A. Sitaram, and M. Sundari

Volume $192 \quad$ No. 2

February 2000 


\title{
HARDY'S UNCERTAINTY PRINCIPLE ON SEMISIMPLE GROUPS
}

\author{
M. Cowling, A. Sitaram, and M. Sundari
}

\begin{abstract}
A theorem of Hardy states that, if $f$ is a function on $\mathbb{R}$ such that $|f(x)| \leq C e^{-\alpha|x|^{2}}$ for all $x$ in $\mathbb{R}$ and $|\hat{f}(\xi)| \leq C e^{-\beta|\xi|^{2}}$ for all $\xi$ in $\mathbb{R}$, where $\alpha>0, \beta>0$, and $\alpha \beta>1 / 4$, then $f=0$. Sitaram and Sundari generalised this theorem to semisimple groups with one conjugacy class of Cartan subgroups and to the $K$-invariant case for general semisimple groups. We extend the theorem to all semisimple groups.
\end{abstract}

\section{Introduction.}

The Uncertainty Principle states, roughly speaking, that a nonzero function $f$ and its Fourier transform $\hat{f}$ cannot both be sharply localised. This fact may be manifested in different ways. The version of this phenomenon described in the abstract is due to Hardy [3]; we call it Hardy's Uncertainty Principle. Considerable attention has been devoted recently to discovering new forms of and new contexts for the Uncertainty Principle (see [2] for a recent comprehensive survey). In particular, Sitaram and Sundari [4] generalised Hardy's Uncertainty Principle to connected semisimple Lie groups with one conjugacy class of Cartan subgroups and to the $K$-invariant case for general connected semisimple Lie groups. We extend the theorem of Sitaram and Sundari [4], and establish a form of Hardy's Uncertainty Principle for all connected semisimple Lie groups with finite centre.

\section{The theorem.}

Let $G$ be a connected real semisimple Lie group with finite centre. Let $K A N$ be an Iwasawa decomposition of $G$, and let $M A N$ be the associated minimal parabolic subgroup of $G$. The Lie algebras of $G$ and $A$ are denoted by $\mathfrak{g}$ and $\mathfrak{a}$. The Killing form of $\mathfrak{g}$ induces an inner product on $\mathfrak{a}$ and hence on the dual $\mathfrak{a}^{*}$; in both cases the corresponding norms are denoted by $|\cdot|$. Haar measures on $K$ and $G$ are fixed; that on $K$ is normalised so that the total mass of $K$ is 1 . Integrals over $G$ and $K$ are relative to these Haar measures.

Any irreducible unitary representation $\mu$ of $M$ may be realised as the lefttranslation representation on a finite-dimensional subspace $\mathcal{H}_{\mu}$ of $C(M)$, the space of continuous complex-valued functions on $M$. For such a $\mu$, and $\lambda$ in 
the complexification $\mathfrak{a}_{\mathbb{C}}^{*}$ of $\mathfrak{a}^{*}$, we define the space $\mathcal{H}_{\mu, \lambda}^{0}$ to be the subspace of $C(G)$ of all functions $\xi$ with the properties that

$$
\xi(\text { gan })=\xi(g) \exp ((i \lambda-\rho) \log a) \quad \forall g \in G \quad \forall a \in A \quad \forall n \in N
$$

and

$$
m \mapsto \xi(g m) \in \mathcal{H}_{\mu} \quad \forall g \in G .
$$

Note that such functions are determined by their restrictions to $K$, i.e., effectively we are dealing with a subspace of $C(K)$. The representation $\pi_{\mu, \lambda}^{0}$ of $G$ is the left-translation representation of $G$ on this space. We define the inner product $\langle\xi, \eta\rangle$ of $\xi$ and $\eta$ in $\mathcal{H}_{\mu, \lambda}^{0}$ to be

$$
\int_{K} \xi(k) \bar{\eta}(k) d k
$$

$\|\cdot\|$ denotes the associated norm.

Denote by $\mathcal{H}_{\mu, \lambda}$ the completion of $\mathcal{H}_{\mu, \lambda}^{0}$ with this norm, and by $\pi_{\mu, \lambda}$ the extension of $\pi_{\mu, \lambda}^{0}$ to $\mathcal{H}_{\mu, \lambda}$. The space $\mathcal{H}_{\mu, \lambda}$ may be identified with a subspace of $L^{2}(K)$, and $\mathcal{H}_{\mu, \lambda}^{0}$ with the space of continuous functions in $\mathcal{H}_{\mu, \lambda}$.

For $\mu$ in $\widehat{M}$ and $\lambda$ in $\mathfrak{a}^{*}$, the representation $\pi_{\mu, \lambda}$ is unitary. This representation lifts to a representation of $L^{1}(G)$ by integration, as follows. First, for $f$ in $L^{1}(G)$ and $\xi$ and $\eta$ in $\mathcal{H}_{\mu, \lambda}$, the integral

$$
\int_{G} f(g)\left\langle\pi_{\mu, \lambda}(g) \xi, \eta\right\rangle d g
$$

converges, to $B_{f}(\xi, \eta)$ say. Next, $B_{f}$ is a sesquilinear form on $\mathcal{H}_{\mu, \lambda}$. Thus there exists a unique bounded operator, denoted $\pi_{\mu, \lambda}(f)$, such that

$$
\left\langle\pi_{\mu, \lambda}(f) \xi, \eta\right\rangle=\int_{G} f(g)\left\langle\pi_{\mu, \lambda}(g) \xi, \eta\right\rangle d g \quad \forall \xi, \eta \in \mathcal{H}_{\mu, \lambda} .
$$

We denote by $\|\cdot\|$ the operator norm of such operators, relative to the given norm on $\mathcal{H}_{\mu, \lambda}$. If $\lambda \in \mathfrak{a}_{\mathbb{C}}^{*} \backslash \mathfrak{a}^{*}$, then the matrix coefficients $g \mapsto\left\langle\pi_{\mu, \lambda}(g) \xi, \eta\right\rangle$ need not be bounded, and for general $f$ in $L^{1}(G)$ it may not be possible to define $\pi_{\mu, \lambda}(f)$. However, for $f$ which decays sufficiently rapidly at infinity in $G$, in particular for $f$ in the theorem below, $\pi_{\mu, \lambda}(f)$ may still be defined by the procedure above.

Theorem. Suppose that $C, \alpha, C_{\mu}, \beta_{\mu}$ are positive constants and $\alpha \beta_{\mu}>1 / 4$ for all $\mu$ in $\widehat{M}$, and that $f$ is a measurable function on $G$ such that

$$
\left|f\left(k a k^{\prime}\right)\right| \leq C \exp \left(-\alpha|\log a|^{2}\right) \quad \forall k, k^{\prime} \in K \quad \forall a \in A
$$

and

$$
\left\|\pi_{\mu, \lambda}(f)\right\| \leq C_{\mu} \exp \left(-\beta_{\mu}|\lambda|^{2}\right) \quad \forall \mu \in \widehat{M} \quad \forall \lambda \in \mathfrak{a}^{*} .
$$

Then $f=0$. 
Proof. Let $\sigma$ and $\tau$ be irreducible representations of $K$, with characters $\chi_{\sigma}$ and $\chi_{\tau}$. Define $f_{\sigma, \tau}$ by the formula

$$
f_{\sigma, \tau}(g)=\operatorname{dim} \sigma \operatorname{dim} \tau \int_{K} \int_{K} \bar{\chi}_{\sigma}(k) \bar{\chi}_{\tau}\left(k^{\prime}\right) f\left(k g k^{\prime}\right) d k d k^{\prime} .
$$

By a straightforward estimate,

$$
\left|f_{\sigma, \tau}\left(k a k^{\prime}\right)\right| \leq C(\operatorname{dim} \sigma \operatorname{dim} \tau)^{2} \exp \left(-\alpha|\log a|^{2}\right) \quad \forall k, k^{\prime} \in K \quad \forall a \in A .
$$

Further, $\pi_{\mu, \lambda}\left(f_{\sigma, \tau}\right)$ is the composition $P_{\sigma} \pi_{\mu, \lambda}(f) P_{\tau}$, where $P_{\sigma}$ and $P_{\tau}$ are the projections of $L^{2}(K)$ onto the $\sigma$-isotypic and $\tau$-isotypic subspaces, so that

$$
\left\|\pi_{\mu, \lambda}\left(f_{\sigma, \tau}\right)\right\| \leq C_{\mu} \exp \left(-\beta_{\mu}|\lambda|^{2}\right) \quad \forall \mu \in \widehat{M} \quad \forall \lambda \in \mathfrak{a}^{*} .
$$

Now the arguments of Section 3 of [4] show that, if $\alpha_{\mu}$ is chosen such that $0<\alpha_{\mu}<\alpha$ and $\alpha_{\mu} \beta_{\mu}>1 / 4$, then

$$
\begin{aligned}
\left\|\pi_{\mu, \lambda}\left(f_{\sigma, \tau}\right)\right\| & \leq C_{\sigma, \tau, \mu} \int_{G} \Phi_{i \operatorname{Re}(\lambda)}(x)|f(x)| d x \\
& \leq C_{\sigma, \tau, \mu}^{\prime} \exp \left(\frac{|\lambda|^{2}}{4 \alpha_{\mu}}\right) \quad \forall \mu \in \widehat{M} \quad \forall \lambda \in a_{\mathbb{C}}^{*},
\end{aligned}
$$

where $\Phi_{i \operatorname{Re}(\lambda)}$ denotes the usual elementary spherical function, and hence that

$$
\pi_{\mu, \lambda}\left(f_{\sigma, \tau}\right)=0 \quad \forall \mu \in \widehat{M} \quad \forall \lambda \in a_{\mathbb{C}}^{*} .
$$

By Harish-Chandra's subquotient theorem (see G. Warner [5, p. 452]), if $\pi$ is any irreducible unitary representation of $G$ on a Hilbert space $\mathcal{H}_{\pi}$, then there exist $\mu$ in $\widehat{M}$ and $\lambda$ in $\mathfrak{a}_{\mathbb{C}}^{*}$ and closed subspaces $S_{0}$ and $S_{1}$ of $\mathcal{H}_{\mu, \lambda}$ such that $\pi$ is Naĭmark equivalent to the quotient representation $\dot{\pi}_{\mu, \lambda}$ of $\pi_{\mu, \lambda}$ on $S_{1} / S_{0}$. This means that there is an intertwining operator $A_{\pi}$ with dense domain and range between $\left(\pi, \mathcal{H}_{\pi}\right)$ and $\left(\dot{\pi}_{\mu, \lambda}, S_{1} / S_{0}\right)$. Consequently $\pi\left(f_{\sigma, \tau}\right)=0$, first on the domain of $A_{\pi}$ by the intertwining property, and then on all $\mathcal{H}_{\pi}$ by continuity. In summary,

$$
\left\langle\pi\left(f_{\sigma, \tau}\right) \xi, \eta\right\rangle=0 \quad \forall \xi, \eta \in \mathcal{H}_{\pi},
$$

and therefore, summing over $\sigma$ and $\tau$, we see that

$$
\langle\pi(f) \xi, \eta\rangle=0 \quad \forall \xi, \eta \in \mathcal{H}_{\pi} .
$$

It follows that $\pi(f)=0$ for all $\pi$ in $\widehat{G}$, the unitary dual of $G$, whence $f=0$ by the Plancherel theorem.

The argument of this paper may also be applied in other contexts. For instance, we may show the following: if $f$ is a measurable function on $G$, rapidly decreasing in the sense that for any $B$ in $\mathbb{R}^{+}$there exists $A$ in $\mathbb{R}^{+}$ such that

$$
\left|f\left(k a k^{\prime}\right)\right| \leq A \exp (-\alpha B|\log a|) \quad \forall k, k^{\prime} \in K \quad \forall a \in A,
$$


and if on each principal series induced from the minimal parabolic subgroup, the group-theoretic Fourier transform vanishes on a set of positive Plancherel measure, then $f$ is zero. This is a qualitative uncertainty principle related to $[\mathbf{1}]$.

\section{References}

[1] M. Cowling, J.F. Price and A. Sitaram, A qualitative uncertainty principle for semisimple Lie groups, J. Austral. Math. Soc. Ser. A, 45 (1988), 127-132.

[2] G.B. Folland and A. Sitaram, The uncertainty principle: a mathematical survey, J. Fourier Anal. Appl., 3 (1997), 207-238.

[3] G.H. Hardy, A theorem concerning Fourier transforms, J. London Math. Soc., 8 (1933), $227-231$.

[4] A. Sitaram and M. Sundari, An analogue of Hardy's theorem for very rapidly decreasing functions on semi-simple Lie groups, Pacific J. Math., 177 (1997), 187-200.

[5] G. Warner, Harmonic Analysis on Semi-Simple Lie Groups I, Grundlehren der math. Wissenschaft, Bd 188, Springer-Verlag, Berlin, Heidelberg, New York, 1972.

Received June 26, 1998. The first and third authors were supported by the Australian Research Council.

University of New South Wales

SYDNEY NSW 2052

Australia

E-mail address: m.cowling@unsw.edu.au

Indian Statistical Institute

BANGALORE - 560059

INDIA

E-mail address: sitaram@isibang.ernet.in

University of New South Wales

SYDNEY NSW 2052

Australia

P.O. Box No. 5978

JEDDAH 21432

Kingdom of Saudi Arabia

E-mail address: madhava@memrbksa.com 